\title{
Constrained Multi-global Optimization using a Penalty Stretched Simulated Annealing Framework
}

\author{
Ana I. Pereira* and Edite M.G.P. Fernandes ${ }^{\dagger}$ \\ ${ }^{*}$ Department of Mathematics, Polytechnic Institute of Bragança, Bragança, Portugal \\ ${ }^{\dagger}$ Department of Production and Systems, University of Minho, 4710-057 Braga, Portugal
}

\begin{abstract}
This paper presents a new simulated annealing algorithm to solve constrained multi-global optimization problems. To compute all global solutions in a sequential manner, we combine the function stretching technique with the adaptive simulated annealing variant. Constraint-handling is carried out through a nondifferentiable penalty function. To benchmark our penalty stretched simulated annealing algorithm we solve a set of well-known problems. Our preliminary numerical results show that the algorithm is promising.
\end{abstract}

Keywords: multi-global optimization, penalty function, simulated annealing

PACS: $02.60 . \mathrm{Pn}$

\section{INTRODUCTION}

The purpose of this paper is to present a penalty framework for solving constrained multi-global optimization (CMGO) problems in the following form

$$
\min _{x} f(x), \text { subject to } g_{j}(x) \leq 0, j=1, \ldots, m \text { and } l_{x} \leq x \leq u_{x}
$$

where at least one of the functions $f, g_{j}: \mathbb{R}^{n} \rightarrow \mathbb{R}$ is nonlinear, and $\mathscr{F}=\left\{x: l_{x} \leq x \leq u_{x}, g_{j}(x) \leq 0, j=1, \ldots, m\right\}$ is the feasible region. Since we do not assume convexity, $f$ may possess many global minima inside $\mathscr{F}$. Here, we aim to find all points $x^{*} \in \mathscr{F}$ such that $f\left(x^{*}\right) \leq f(x)$ for all $x \in \mathscr{F}$. We also assume that the problem (1) has a finite number of global minimizers. This class of global optimization problems is very important and frequently encountered in engineering applications (e.g. [2, 3, 4]). Some algorithms for solving this type of problem require substantial gradient information and aim to improve the solution in a neighborhood of a given initial approximation. When the problem has more than one global solution, the probability of convergence to an already detected global solution is very high, and depends very closely on the provided initial approximation.

The most well-known category of methods to handle constraints in nonlinear optimization problems depends on a penalty function and a positive penalty parameter. Techniques based on penalty functions transform the constrained problem into a sequence of unconstrained problem by penalizing $f$ when constraints are violated and then minimizing the penalty function using methods for unconstrained problems. It is expected that the penalty parameter is updated along the iterative process, so that convergence to the solution can be accelerated. The choice of the initial penalty and its updating formula are issues not well-defined until now. Large values give feasible solutions that have low accuracy, since search around the boundary tends to be avoided, while small values generate infeasible with good accuracy solutions.

The remainder of this paper is organized as follows. In the next section, we briefly introduce the penalty function that is used to penalize infeasible iterates. Then, we describe the implementation of the function stretching technique coupled with the simulated annealing based algorithm so that all global solutions can be computed in a sequential manner. The last sections contain the results of all the numerical experiments and the conclusions. 


\section{CONSTRAINT-HANDLING BY A PENALTY TECHNIQUE}

The basic penalty approach defines a fitness for each point $x$, herein denoted by $\phi(x ; \mu)$, by adding to the objective function value a penalty term that aims to penalize infeasible solutions as follows:

$$
\phi(x ; \mu)=f(x)+\mu \sum_{j=1}^{m}\left(\max \left\{0, g_{j}(x)\right\}\right)^{\gamma\left(g_{j}(x)\right)},
$$

where $\mu$ is the positive penalty parameter. The power of the constraint violation, $\gamma($.$) , is a violation dependent constant:$ $\gamma(z)=1$ if $z \leq 0.1$, and $\gamma(z)=2$, otherwise. See, for example, in [7, 8]. We remark that only the inequality constraints are used in the penalized terms. It follows that the minimizer of the function $\phi$, in (2), that satisfies $l_{x} \leq x \leq u_{x}$ for a fixed value of the parameter $\mu$, herein denoted by $x^{*}(\mu)$, converges to the solution of the given problem (1) as $\mu$ increases [1]. An appropriate updating scheme for $\mu$ is as follows:

$$
\mu_{k+1}=\max \left\{\tau \mu_{k}, \mu_{\max }\right\}, \text { for } \tau>1 \text { and } \mu_{\max }>>1
$$

for a given $\mu_{0}>0$, where $k$ represents the iteration counter.

\section{PENALTY STRETCHED SIMULATED ANNEALING METHOD}

Here, we use the following notation: $N$ is the number of global solutions of problem (1), $X^{*}=\left[x_{1}^{*}, x_{2}^{*}, \ldots, x_{N}^{*}\right]$ is the $n \times N$ matrix whose columns contain the global solutions. In general, each implementation of a global optimization method finds just one global solution. To be able to compute multiple solutions, deflation techniques have to be incorporated in the algorithm. Our proposal relies on a sequential simulated annealing (SA) algorithm in the sense that a sequence of global optimization problems is iteratively defined and each problem is solved by the SA algorithm, a global point-to-point stochastic algorithm. After the computation of a global solution, the objective function is transformed using a function stretching technique.

$>$ From now on and to simplify the notation, we use $\phi(x)$ instead of $\phi(x ; \mu)$. The function stretching technique was initially proposed in [9], in a particle swarm optimization algorithm context, to provide a way to escape from a local solution, driving the search to a global one. When a local (non-global) solution, $\widehat{x}$, is found, the technique stretches the function $\phi$ by augmenting (by a certain amount) the objective function at all points $x$ that satisfy $\phi(x)>\phi(\widehat{x})$, leaving $\phi(x)$ unchanged for all $x$ such that $\phi(x) \leq \phi(\hat{x})$. The minimization process is then repeated with the new stretched objective function to look for the global solution. If another local solution is found, the previously mentioned process is repeated. Each global optimization problem of the sequence is solved by ASA, a well-known variant of the simulated annealing algorithm [5].

In our case, the function stretching technique aims to prevent the convergence of the SA algorithm to a previously found solution. Thus, the function stretching technique is applied only locally, in order to transform $\phi(x)$ in a neighborhood of $x_{l}^{*}$, say $V_{\varepsilon}\left(x_{l}^{*}\right), \varepsilon>0$. So, $\phi(x)$ is augmented only on the region $V_{\varepsilon}\left(x_{l}^{*}\right)$ leaving all the other minima unchanged. The minimum $\phi\left(x_{l}^{*}\right)$ disappears but all other minima are left unchanged. Each global optimization problem of the sequence is solved by ASA (adaptive simulated annealing), a well-known variant of the simulated annealing algorithm [5]. The multi-global procedure terminates when for a predefined set of consecutive iterations no other solution is encountered [11, 12].

This sequential simulated annealing algorithm solves a sequence of global optimization problems whose objective function is obtained by applying a function stretching technique, as outlined in $[9,10]$, to the objective function of the previous problem in the sequence. The mathematical formulation of the algorithm together with the transformations that are carried out are the following:

$$
\min _{l_{x} \leq x \leq u_{x}} \Phi_{l}(x) \equiv \begin{cases}\hat{\phi}(x) & \text { if } x \in V_{\varepsilon^{l}}\left(x_{l}^{*}\right), l \in\{1, \ldots, N\} \\ \phi(x) & \text { otherwise }\end{cases}
$$

where $\hat{\phi}(x)$ is defined as

$$
\hat{\phi}(x)=\bar{\phi}(x)+\frac{\delta_{2}\left[\operatorname{sgn}\left(\phi(x)-\phi\left(x_{l}^{*}\right)\right)+1\right]}{2 \tanh \left(\kappa\left(\bar{\phi}(x)-\bar{\phi}\left(x_{l}^{*}\right)\right)\right.}
$$

and

$$
\bar{\phi}(x)=\phi(x)+\frac{\delta_{1}}{2}\left\|x-x_{l}^{*}\right\|\left[\operatorname{sgn}\left(\phi(x)-\phi\left(x_{l}^{*}\right)\right)+1\right]
$$


with $\delta_{1}, \delta_{2}$ and $\kappa$ positive constants and "sgn" defines the well-known sign function. Transformations (5) and (4) stretch the neighborhood of $x_{l}^{*}$, with ray $\varepsilon^{l}$, upwards assigning larger function values to those points to prevent the convergence of the global optimization method to that computed solution [12]. The proposed penalty stretched simulated annealing (PSSA) algorithm for CMGO is presented below:

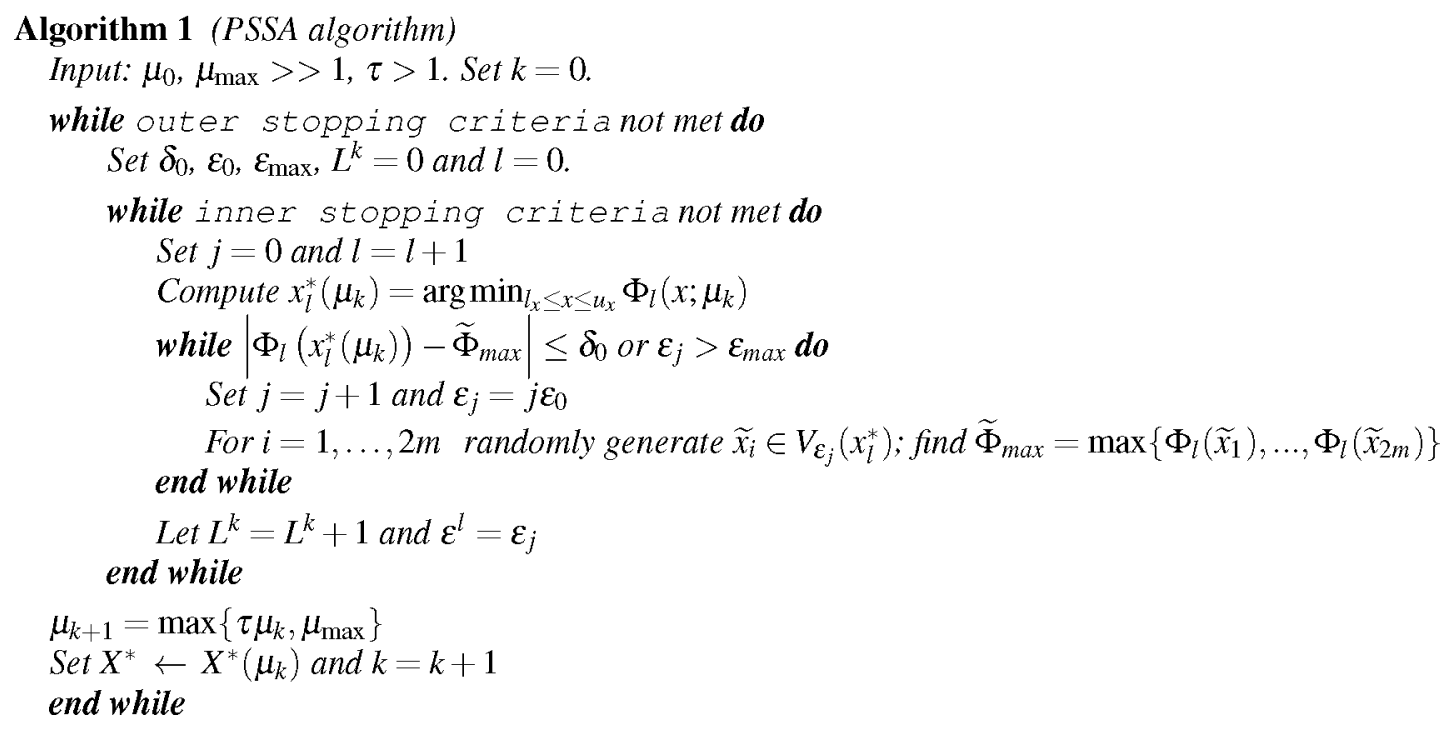

In the PSSA algorithm, the conditions to stop the outer iterative process are: $\left\|X^{*}\left(\mu_{k}\right)-X^{*}\left(\mu_{k-1}\right)\right\| \leq \varepsilon_{x}$ or $k>k_{\max }$. The inner iterative process terminates if $L^{k}$ does not change for a specified $\mathscr{K}$ iterations.

\section{NUMERICAL RESULTS}

The herein proposed multi-global optimization method based on a penalty technique for constraint-handling was implemented in the C programming language on a Pentium II, Celeron $466 \mathrm{Mhz}$ with $64 \mathrm{Mb}$ of RAM. To evaluate the performance of the herein proposed penalty stretched simulated annealing algorithm for constrained multi-global optimization problems a set of six benchmark problems, described in full detail in the Appendix of [13], is used. In this preliminary study, small dimensional problems ( $n \leq 10$ and $m \leq 13$ ) with a nonlinear objective function, simple bounds and inequality constraints were tested. They are known in the literature as g04, g06, g08, g09, g12 and g18.

The values for the user defined parameters are: $\tau=10, \mu_{\max }=10^{8}, \mu_{0}=10, \delta_{0}=10^{-3}, \varepsilon_{0}=0.15, \varepsilon_{\max }=5$, $k_{\max }=10$ and $\varepsilon_{x}=10^{-3}$, and $\mathscr{K}=5$. Each problem was solved by PSSA five times with randomly generated initial approximations.

Details of the selected problems are listed in the Table 1, where $\mathbf{P}$ refers to the problem number, "type of $f^{\prime \prime}$ describes the type of objective function, $f_{g l o b a l}$ is the known global minimum, $n$ is the number of variables, $m$ is the number of inequality constraints, $f_{P S S A}^{*}$ is our best solution obtained after the five runs, $\bar{f}_{P S S A}$ is the average of the obtained solutions over the five runs, $\mathbf{N}_{P S S A}$ is the average number of iterations reuired by the penalty stretched simulated annealing method, $\mathbf{N}_{f e v a l}$ is the average number of function evaluations and $\mathbf{N}_{l o c}$ gives the average of the obtained global/local solutions.

TABLE 1. Numerical results

\begin{tabular}{lcccc|ccccc}
\hline $\mathbf{P}$ & type of $f$ & \multicolumn{1}{c}{$f_{\text {global }}$} & $n$ & $m$ & $f_{P S S A}^{*}$ & $\bar{f}_{\text {PSSA }}$ & $\mathbf{N}_{\text {PSSA }}$ & $\mathbf{N}_{\text {feval }}$ & $\mathbf{N}_{\text {loc }}$ \\
\hline g04 & quadratic & $-3.0665 \mathrm{E}+04$ & 5 & 6 & $-2.5874 \mathrm{E}+04$ & $-2.5874 \mathrm{E}+04$ & 43 & 156154 & 12 \\
g06 & cubic & $-6.9618 \mathrm{E}+03$ & 2 & 2 & $-6.9419 \mathrm{E}+03$ & $-6.9419 \mathrm{E}+03$ & 9 & 27550 & 1 \\
g08 & general & $-9.5825 \mathrm{E}-02$ & 2 & 2 & $-9.5825 \mathrm{E}-02$ & $-8.3552 \mathrm{E}-02$ & 24 & 79771 & 5 \\
g09 & general & $6.8063 \mathrm{E}+02$ & 7 & 4 & $6.8870 \mathrm{E}+02$ & $6.8875 \mathrm{E}+02$ & 6 & 309719 & 1 \\
g12 & quadratic & $1.0000 \mathrm{E}+00$ & 3 & 1 & $1.0000 \mathrm{E}+00$ & $9.8562 \mathrm{E}-01$ & 24 & 202219 & 1 \\
g18 & quadratic & $-8.6603 \mathrm{E}-01$ & 9 & 13 & $-8.6349 \mathrm{E}-01$ & $-8.5987 \mathrm{E}-01$ & 10 & 945000 & 5 \\
\hline
\end{tabular}




\section{CONCLUSIONS}

We have incorporated a penalty framework into a sequential simulated annealing algorithm that uses a function stretching technique, to be able to compute sequentially all the global solutions of inequality constrained multi-modal objective based optimization problems.

The preliminary numerical tests with a set of small problems are encouraging. Further experiments, in particular with problems with a large number of local solutions, and with practical engineering applications are required. To improve solution accuracy, the use of a local search to refine the obtained solution was mandatory.

Future challenge is the use of other penalty functions and the extension of this type of multi-global method to equality constrained problems.

\section{ACKNOWLEDGMENTS}

This work has been partially supported by the FCT (Fundação para a Ciência e a Tecnologia), Portugal.

\section{REFERENCES}

1. D. P. Bertsekas, Constrained Optimization and Lagrange Multiplier Methods, Academic Press, 1982.

2. A. Bonilla-Petriciolet, R. Vázquez-Román, G. A. Iglesias-Silva, K. R. Hall. Performance of Stochastic Global Optimization Methods in the Calculation of Phase Analyses for Nonreactive and Reactive Mixtures. Industrial Engineering Chemistry Research, 45, 4764-4772 (2006).

3. C. M. McDonald, C. A. Floudas, Global optimization for the phase stability problem, AIChE. J., 41, 1798-1814 (1995).

4. C. Floudas, Recent advances in global optimization for process synthesis, design and control: enclosure all solutions, Computers and Chemical Engineering 963, 963-973 (1999).

5. L. Ingber, Very fast simulated re-annealing, Mathematical and Computer Modelling, 12, 967-973 (1989).

6. T. León, S. Sanmatias and E. Vercher, A multilocal optimization algorithm, TOP 6 (1998), 1-18.

7. J.-L. Liu and J.-H. Lin, Evolutionary computation of unconstrained and constrained problems using a novel momentum-type particle swarm optimization, Engineering Optimization, 39(3), 287-305, 2007.

8. Y.G. Petalas, K.E. Parsopoulos and M.N. Vrahatis, Memetic particle swarm optimization, Annals of Operations Research, $156,99-127,2007$.

9. K. Parsopoulos, V. Plagianakos, G. Magoulas, and M. Vrahatis, Objective function stretching to alleviate convergence to local minima, Nonlinear Analysis 47, 3419-3424 (2001).

10. K. Parsopoulos, and M. Vrahatis, Recent approaches to global optimization problems through particle swarm optimazition, Natural Computing 1, 235-306 (2002).

11. A. I. P. N. Pereira and E. M. G. P. Fernandes, "On a reduction line search filter method for nonlinear semi-infinite programming problems", in Euro Mini Conference "Continuous Optimization and Knowledge-Based Technologies", edited by L. Sakalauskas, G.W. Weber, E.K. Zavadskas, ISBN: 978-9955-28-283-9, 2008, pp. 174-179.

12. A. I. P. N. Pereira and E. M. G. P. Fernandes, A reduction method for semi-infinite programming by means of a global stochastic approach, Optimization, 58, 713-726 (2009).

13. A. E. M. Zavala, A. H. Aguirre, and E. R. V. Diharce. Constrained optimization via particle evolutionary swarm optimization algorithm (PESO). In GECCO'05, 209-216, 2005. 